\title{
Correlation of prechemotherapy urinary megalin ectodomain (A-megalin) levels with the development of cisplatin-induced nephrotoxicity: a prospective observational study
}

Satoshi Shoji ${ }^{1}$, Michihiro Hosojima ${ }^{2^{*}}$ (D, Hideyuki Kabasawa ${ }^{2}$, Rie Kondo ${ }^{1}$, Satoru Miura ${ }^{1,3}$, Satoshi Watanabe ${ }^{1}$ Nobumasa Aoki ${ }^{1}$, Ryohei Kaseda ${ }^{4}$, Shoji Kuwahara ${ }^{5,6}$, Naohito Tanabe ${ }^{7}$, Yoshiaki Hirayama ${ }^{8}$, Ichiei Narita ${ }^{4}$, Toshiaki Kikuchi ${ }^{1}$, Hiroshi Kagamu ${ }^{1,9}$ and Akihiko Saito ${ }^{5^{*}}$

\begin{abstract}
Background: Cisplatin is a potent chemotherapeutic agent used to treat a variety of solid tumors. One of the major side effects of cisplatin is dose-limiting nephrotoxicity. We recently demonstrated that the renal uptake of cisplatin and resultant cisplatin-induced nephrotoxicity are mediated in part by megalin, an endocytic receptor in proximal tubule epithelial cells (PTECs). We also developed sandwich enzyme-linked immunosorbent assays to measure the megalin ectodomain (A-megalin) and full-length megalin (C-megalin) in urine using monoclonal antibodies against the aminoand carboxyl-termini of megalin, respectively. The present study examined the correlation of urinary megalin level with cisplatin-induced nephrotoxicity and its utility as a biomarker in patients with thoracic cancer.
\end{abstract}

Methods: This prospective observational study involved 45 chemotherapy-naive patients scheduled to receive chemotherapy with $\geq 60 \mathrm{mg} / \mathrm{m}^{2}$ cisplatin for histologically diagnosed small cell lung cancer, non-small cell lung cancer, or malignant pleural mesothelioma. Before and after the first course of chemotherapy, we measured urinary A- and Cmegalin and other markers of PTEC injury, such as N-acetyl- $\beta$-D-glucosaminidase, $a_{1}$-microglobulin, $\beta_{2}$-microglobulin, neutrophil gelatinase-associated lipocalin, and liver-type fatty acid-binding protein, and compared the values with the change in the estimated glomerular filtration rate (eGFR) and clinical risk factors for renal impairment.

Results: A negative correlation was found between baseline urinary A-megalin levels and change in eGFR ( $r=-0.458$, $P=0.002$ ). According to Kaplan-Meier survival curves, eGFR decline was associated with the baseline urinary A-megalin quartile ( $P=0.038$ ). In addition, according to the hazard ratios (HRs) for eGFR decline $>10 \mathrm{~mL} / \mathrm{min} / 1.73 \mathrm{~m}^{2}$ calculated using a Cox proportional hazard model, the highest quartile had a significantly higher risk of eGFR decline compared with the lowest quartile (HR 7.243; 95\% confidence interval 1.545-33.962). Other baseline urinary markers showed no correlation with eGFR decline.

(Continued on next page)

\footnotetext{
*Correspondence: hos09582@med.niigata-u.ac.jp; akisaito@med.niigatau.ac.jp

${ }^{2}$ Department of Clinical Nutrition Science, Kidney Research Center, Niigata University Graduate School of Medical and Dental Sciences, 1-757 Asahimachi-dori, Chuo-ku, Niigata, Niigata 951-8510, Japan

${ }^{5}$ Department of Applied Molecular Medicine, Kidney Research Center, Niigata

University Graduate School of Medical and Dental Sciences, 1-757

Asahimachi-dori, Chuo-ku, Niigata, Niigata 951-8510, Japan

Full list of author information is available at the end of the article
}

(c) The Author(s). 2019 Open Access This article is distributed under the terms of the Creative Commons Attribution 4.0 International License (http://creativecommons.org/licenses/by/4.0/), which permits unrestricted use, distribution, and reproduction in any medium, provided you give appropriate credit to the original author(s) and the source, provide a link to the Creative Commons license, and indicate if changes were made. The Creative Commons Public Domain Dedication waiver (http://creativecommons.org/publicdomain/zero/1.0/) applies to the data made available in this article, unless otherwise stated. 
(Continued from previous page)

Conclusions: This is the first report demonstrating that prechemotherapy urinary A-megalin levels are correlated with the development of cisplatin-induced nephrotoxicity. This finding has clinical implications for the identification of patients at risk for cisplatin-induced nephrotoxicity and the development of possible prophylactic therapies.

Keywords: Chemotherapy, Cisplatin, Nephrotoxicity, Urinary megalin

\section{Background}

Cisplatin (cis-dichlorodiammineplatinum [II]) is one of the most potent chemotherapeutic agents used in the treatment of various solid tumors, including bladder cancer, cervical cancer, malignant pleural mesothelioma (MPM), ovarian cancer, squamous cell carcinoma of the head and neck, germ cell cancer, small cell lung cancer (SCLC), and non-small cell lung cancer (NSCLC). Cisplatin chemotherapy in combination with radiotherapy or surgery improves survival in advanced lung cancer and cure rate in locoregional and early stage lung cancer $[1,2]$.

The dose-limiting toxicity of cisplatin is renal, rather than hematological, typically causing acute kidney injury or even chronic renal impairment [3]. Cisplatin is thus contraindicated for chemotherapy in patients with chronic kidney disease, despite its outstanding efficacy. The kidneys are particularly vulnerable to toxic insult by cisplatin because of its high levels of accumulation in renal tissue [4]. Proximal tubule epithelial cells (PTECs) have been recognized as the primary target of cisplatininduced toxicity [4]. Candidates for facilitated transport systems associated with cisplatin-induced nephrotoxicity include organic cation transporter 2 and copper transporter 1 in PTECs $[5,6]$. However, an organic cation transporter 2 inhibitor, cimetidine, has shown only a partial protective effect against cisplatin-induced nephrotoxicity [5]. Thus, it is important to elucidate the precise mechanism underlying cisplatin-induced nephrotoxicity and develop strategies for its prediction and prevention.

Megalin is a large $(\sim 600 \mathrm{kDa})$ glycoprotein member of the low-density lipoprotein receptor family [7] that is expressed at the apical membranes of PTECs [8]. Megalin plays a pivotal role in the tubular reabsorption of glomerular filtrates and mediates intracellular signal transduction [9]. A high-fat-diet-induced mouse model of diabetic kidney disease showed that megalin mediates the proximal tubular uptake of nephrotoxic substances, such as lipidmodified proteins, resulting in tubuloglomerular alterations [10]. Megalin also mediates the uptake of nephrotoxic drugs such as aminoglycosides [11, 12], polymyxin B [11], colistin [12, 13], and vancomycin [12]. We demonstrated that cisplatin is a ligand of megalin using quartz crystal microbalance analysis. Moreover, we showed that PTECs expressing megalin develop specific cisplatin-induced injury in a mosaic megalin knockout mouse model, but that
PTECs lacking megalin do not [12]. Therefore, megalin likely plays a primary role in the development of cisplatininduced nephrotoxicity.

We also reported that two forms of megalin are excreted in urine, namely, the ectodomain (A-megalin) and full-length (C-megalin) forms, and that the former is several 100-fold more abundant than the latter [14]. We developed sandwich enzyme-linked immunosorbent assays (ELISAs) to measure urinary A- and C-megalin by using monoclonal antibodies (mAbs) against the aminoand carboxyl-termini of megalin, respectively [14]. Urinary $\mathrm{C}$-megalin levels are increased via exocytosis from residual functioning nephrons that are overloaded by megalin-mediated protein metabolism [15]. In contrast, urinary A-megalin excretion appears to be regulated by the intracellular recycling [16] and intramembrane proteolysis [17-19] of megalin.

In this study, we measured urinary megalin levels, focusing in particular on A-megalin, in patients with advanced thoracic malignancies who were treated with cisplatin $\left(\geq 60 \mathrm{mg} / \mathrm{m}^{2}\right)$, with the aim of investigating the pathological role of megalin in the development of cisplatin-induced nephrotoxicity and elucidating the potential of urinary megalin measurement as a biomarker to predict nephrotoxicity.

\section{Methods \\ Patients}

Forty-five consecutive chemotherapy-naive patients with Eastern Cooperative Oncology Group performance status of $0-1$ and intact renal function (estimated glomerular filtration rate $[\mathrm{eGFR}]>60 \mathrm{~mL} / \mathrm{min} / 1.73 \mathrm{~m}^{2}$ ) who were scheduled to receive chemotherapy with $\geq 60 \mathrm{mg} /$ $\mathrm{m}^{2}$ cisplatin for histologically diagnosed NSCLC, SCLC, or MPM were enrolled at a single institution (Niigata University Medical and Dental Hospital, Niigata, Japan). This study was conducted in accordance with the Declaration of Helsinki and Good Clinical Practice guidelines and was approved by the Niigata University Ethics Committee. The patients were enrolled after providing written informed consent.

\section{Study design}

On the day of the first course of chemotherapy (day 0), we obtained baseline serum creatinine $(\mathrm{Cr})$ concentrations and urinary levels of A-megalin, C-megalin, $N$-acetyl- $\beta$-D- 
glucosaminidase (NAG), $\alpha_{1}$-microglobulin $\left(\alpha_{1}-\mathrm{MG}\right), \beta_{2^{-}}$ microglobulin $\left(\beta_{2}-\mathrm{MG}\right)$, neutrophil gelatinase-associated lipocalin (NGAL), liver-type fatty acid-binding protein (LFABP), and Cr. All patients received an intravenous infusion of $\geq 1500 \mathrm{~mL}$ isotonic electrolyte solution, supplemented with magnesium sulfate and diuretics (furosemide or mannitol). After antiemetic therapy consisting of 0.75 $\mathrm{mg}$ palonosetron, $9.9 \mathrm{mg}$ dexamethasone, and the oral administration of $125 \mathrm{mg}$ aprepitant, the patients received a 1-h intravenous infusion of cisplatin, followed by $1000 \mathrm{~mL}$ isotonic electrolyte solution. Fresh urine samples $(20 \mathrm{~mL})$ were scheduled to be collected on day 0 in the morning before cisplatin administration, and on days $1,2,4,6,7$, 12 , and 20 after administration. Urine samples were generally collected on the scheduled day \pm 1 or 2 days. Further urine sampling and measurement of biomarkers were performed at the discretion of the attending physicians. Urine samples were frozen at $-80^{\circ} \mathrm{C}$ and thawed immediately prior to analysis. Serum $\mathrm{Cr}$ concentrations were routinely monitored after cisplatin administration (day 0 in the morning before cisplatin administration, and on days 2,4 , 7, 9, and 12 after administration). Serum $\mathrm{Cr}$ was generally

Table 1 Baseline characteristics of all study patients $(n=45)$

\begin{tabular}{|c|c|c|c|c|c|c|}
\hline & All & Q1 & Q2 & Q3 & Q4 & $P$ \\
\hline & $n=45$ & $n=11$ & $n=12$ & $n=11$ & $n=11$ & \\
\hline \multicolumn{7}{|l|}{ Urinary A-megalin (pmol/g Cr) } \\
\hline Mean \pm SD or [Median] & $87.9 \pm 46.6$ & {$[36.1]$} & {$[76.0]$} & {$[95.8]$} & {$[150.4]$} & \\
\hline Range & $1.6-203.6$ & $1.6-52.9$ & $68.3-79.8$ & $79.9-118.9$ & 119.8-203.6 & \\
\hline \multicolumn{7}{|l|}{ Age, years } \\
\hline Mean \pm SD & $64.6 \pm 8.2$ & $62.1 \pm 8.9$ & $65.8 \pm 4.9$ & $65.5 \pm 8.4$ & $64.8 \pm 10.3$ & 0.702 \\
\hline Sex, $n(\%)$ & & & & & & 0.399 \\
\hline Female & $9(20.0)$ & $3(27.3)$ & $4(33.3)$ & $1(9.1)$ & $1(9.1)$ & \\
\hline Male & $36(80.0)$ & $8(72.7)$ & $8(66.7)$ & $10(90.9)$ & $10(90.9)$ & \\
\hline \multicolumn{7}{|l|}{ Body height, $\mathrm{cm}$} \\
\hline Mean \pm SD & $163.9 \pm 7.2$ & $165.0 \pm 6.9$ & $161.3 \pm 6.0$ & $165.2 \pm 7.0$ & $164.5 \pm 8.9$ & 0.528 \\
\hline \multicolumn{7}{|l|}{ Body weight, kg } \\
\hline Mean \pm SD & $57.7 \pm 10.1$ & $60.2 \pm 10.2$ & $59.9 \pm 12.8$ & $54.3 \pm 10.6$ & $56.0 \pm 4.9$ & 0.437 \\
\hline Smoking status, $n(\%)$ & & & & & & 0.527 \\
\hline Current/former & $40(88.9)$ & $10(90.9)$ & $10(83.3)$ & $11(100)$ & $9(81.8)$ & \\
\hline Never & $5(11.1)$ & $1(9.1)$ & $2(16.7)$ & $0(0.0)$ & $2(18.2)$ & \\
\hline \multicolumn{7}{|l|}{ Baseline therapies, $n(\%)$} \\
\hline RAS inhibitors & $10(22.2)$ & $3(27.3)$ & $2(16.7)$ & $4(36.3)$ & $1(9.1)$ & 0.457 \\
\hline NSAIDs & $22(48.9)$ & $5(45.5)$ & $5(45.5)$ & $4(36.3)$ & $8(72.7)$ & 0.340 \\
\hline \multicolumn{7}{|l|}{ Baseline comorbidity, n (\%) } \\
\hline Hypertension & $20(44.0)$ & $3(27.3)$ & $9(75.0)$ & $5(45.5)$ & $3(27.3)$ & 0.066 \\
\hline Diabetes & $9(20.0)$ & $1(9.1)$ & $2(16.7)$ & $3(27.3)$ & $3(27.3)$ & 0.675 \\
\hline Initial eGFR (mL/min/1.73 $\left.\mathrm{m}^{2}\right)$ & & & & & & 0.372 \\
\hline Mean \pm SD & $89.7 \pm 15.9$ & $83.7 \pm 16.2$ & $87.9 \pm 17.1$ & $92.9 \pm 17.6$ & $94.6 \pm 11.9$ & \\
\hline$\geqq 90$ & $24(53.3)$ & $4(36.4)$ & $8(66.7)$ & $6(54.5)$ & $6(54.5)$ & \\
\hline $60-89$ & $20(44.4)$ & $7(63.6)$ & $3(25.0)$ & $5(45.5)$ & $5(45.5)$ & \\
\hline$\leqq 59$ & $1(2.2)$ & $0(0.0)$ & $1(8.3)$ & $0(0.0)$ & $0(0.0)$ & \\
\hline \multicolumn{7}{|l|}{ Cisplatin dose $\left(\mathrm{mg} / \mathrm{m}^{2}\right)$} \\
\hline Mean \pm SD & $76.0 \pm 5.0$ & $73.2 \pm 6.8$ & $77.5 \pm 2.6$ & $75.9 \pm 5.8$ & $77.3 \pm 2.6$ & 0.143 \\
\hline \multicolumn{7}{|l|}{ Type of malignancy, $n$ (\%) } \\
\hline NSCLC & 33 & & & & & \\
\hline SCLC & 10 & & & & & \\
\hline MPM & 2 & & & & & \\
\hline
\end{tabular}


collected on the scheduled day \pm 1 or 2 days. An adverse renal event was defined as eGFR decline $>10 \mathrm{~mL} / \mathrm{min} /$ $1.73 \mathrm{~m}^{2}$. This definition was based on a report that eGFR levels decrease by approximately $10 \mathrm{~mL} / \mathrm{min} / 1.73 \mathrm{~m}^{2}$ on average for each cycle of cisplatin treatment in cancer patients [20]. Smoking status and baseline therapies, using either renin-angiotensin system (RAS) inhibitors or nonsteroidal anti-inflammatory drugs (NSAIDs), were confirmed from medical records. The presence of hypertension and diabetes was determined by clinical diagnosis.

\section{Measurement of human megalin in urine}

Urinary A- and C-megalin were measured by ELISAs in the laboratory of Denka Co., Ltd., as described previously [14]. In brief, the capture mAbs $(5 \mathrm{mg} / \mathrm{mL})$ were immobilized on ELISA plates (LumiNunc F16 Maxisorp
Surface Plate; Thermo Fisher Scientific, Inc., Waltham, MA) at $4{ }^{\circ} \mathrm{C}$ overnight. The Fab' fragments of the tracer mAbs were conjugated to alkaline phosphatase (Roche Diagnostics, GmbH, Mannheim, Germany). Urine samples $(90 \mu \mathrm{L})$ were mixed with $10 \mu \mathrm{L}$ solution A $(2 \mathrm{~mol} / \mathrm{L}$ Tris- $\mathrm{HCl}, \quad 0.2 \mathrm{~mol} / \mathrm{L}$ ethylenediaminetetraacetic acid, $10 \%$ Triton X-100; $\mathrm{pH} 8.0$ ) and incubated for 1 min at room temperature for the C-megalin assay, or for $3 \mathrm{~h}$ at $50^{\circ} \mathrm{C}$ for the A-megalin assay, and reacted with alkaline phosphatase-labeled tracer mAbs in the ELISA plates. Urinary megalin concentrations were standardized by adjustment to urinary $\mathrm{Cr}$ concentrations.

\section{Measurement of other markers}

Plasma concentrations of $\mathrm{Cr}$ were measured by an enzymatic method in the clinical laboratory at Niigata

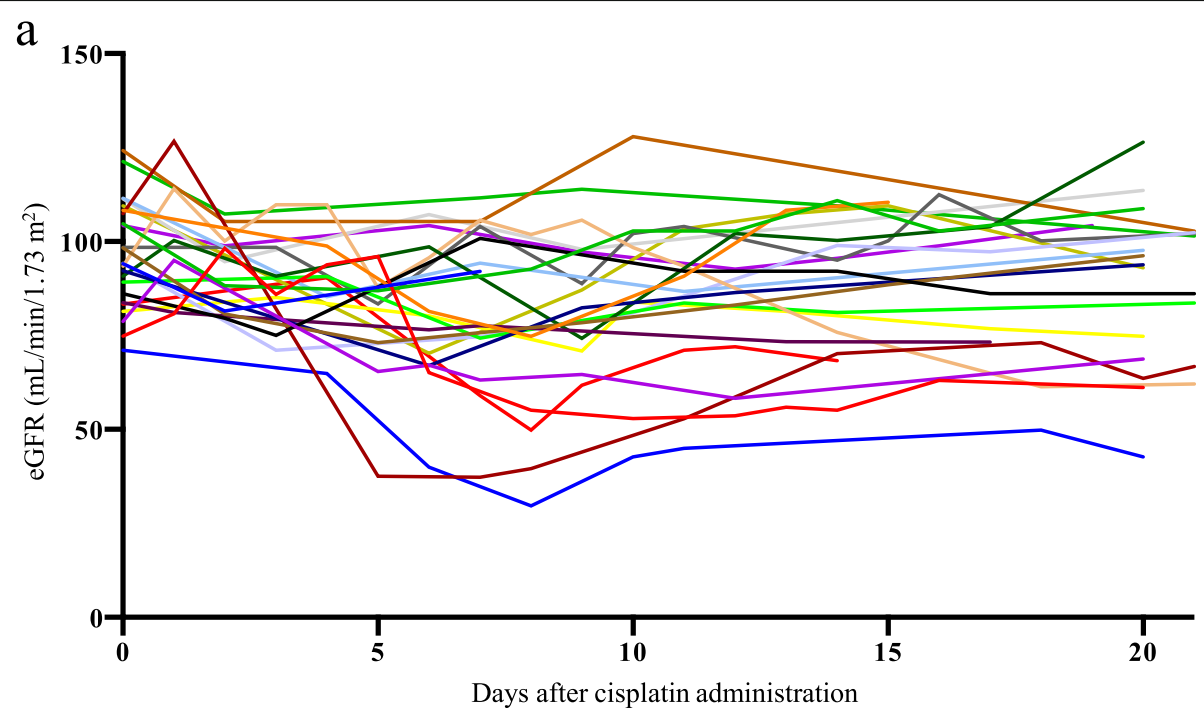

b

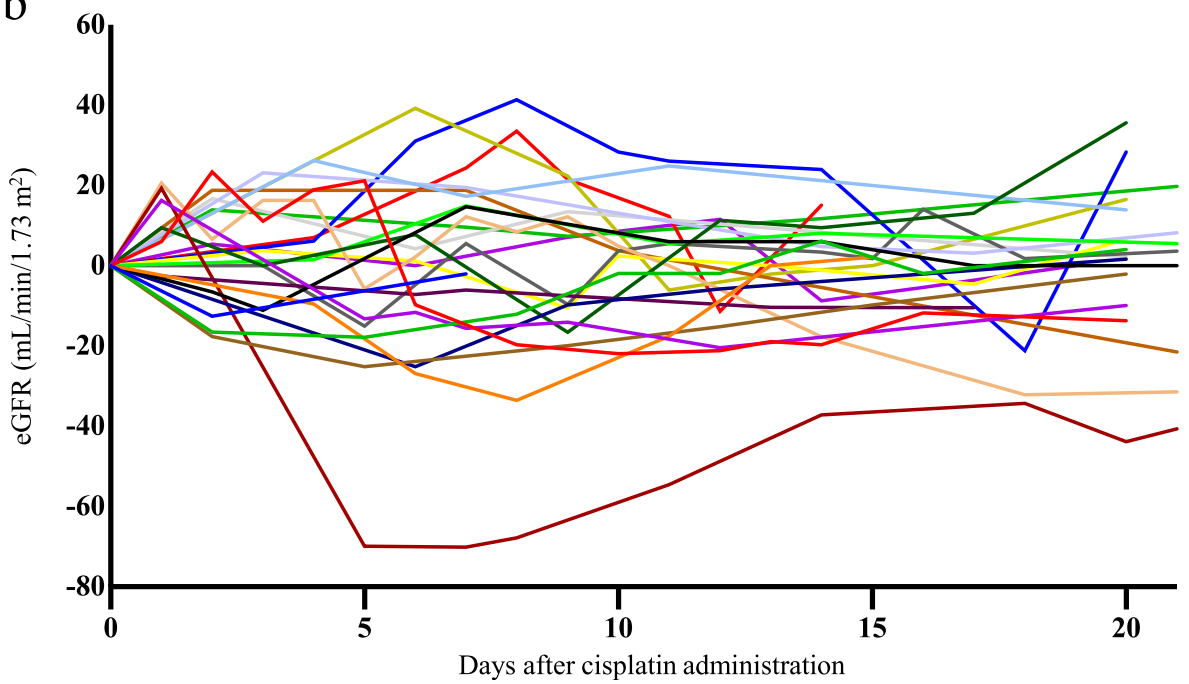

Fig. 1 eGFR levels after cisplatin administration in 24 cases with an adverse renal event. The vertical axis represents the absolute value of eGFR (a) and the change from baseline eGFR (b), respectively 
Table 2 Correlations between renal function and urinary markers

\begin{tabular}{|c|c|c|c|c|}
\hline & \multicolumn{2}{|l|}{ eGFR } & \multicolumn{2}{|l|}{$\Delta \mathrm{eGFR}$} \\
\hline & $r$ & $P$ & $r$ & $P$ \\
\hline eGFR & - & & -0.395 & 0.007 \\
\hline$\Delta \mathrm{eGFR}$ & -0.395 & 0.007 & - & \\
\hline A-megalin & 0.299 & 0.046 & -0.458 & 0.002 \\
\hline$\Delta \mathrm{A}$-megalin & 0.241 & 0.111 & -0.242 & 0.110 \\
\hline C-megalin & 0.043 & 0.780 & -0.208 & 0.171 \\
\hline$\Delta C$-megalin & 0.075 & 0.623 & -0.179 & 0.239 \\
\hline NAG & -0.260 & 0.864 & -0.142 & 0.352 \\
\hline$\triangle N A G$ & -0.092 & 0.546 & -0.235 & 0.12 \\
\hline$a_{1}-M G$ & -0.065 & 0.669 & -0.287 & 0.056 \\
\hline$\Delta \mathrm{a}_{1}-\mathrm{MG}$ & -0.023 & 0.880 & 0.231 & 0.126 \\
\hline$\beta_{2}-M G$ & -0.142 & 0.352 & -0.048 & 0.757 \\
\hline$\Delta \beta_{2}-\mathrm{MG}$ & -0.248 & 0.100 & -0.038 & 0.802 \\
\hline NGAL & -0.071 & 0.642 & 0.124 & 0.417 \\
\hline L-FABP & 0.123 & 0.421 & -0.074 & 0.629 \\
\hline
\end{tabular}

$r$, Pearson's correlation coefficient. $a_{1}-M G, a_{1}$-microglobulin; $\beta_{2}-M G, \beta_{2}-$ microglobulin; eGFR, estimated glomerular filtration rate; L-FABP, liver-type fatty acid-binding protein; NAG, $N$-acetyl- $\beta$-D-glucosaminidase; NGAL, neutrophil gelatinase-associated lipocalin. $\triangle \mathrm{eGFR}$, maximum change from the baseline value to the lowest value of eGFR during follow-up. $\Delta$ urinary marker $=$ (maximum urinary marker after cisplatin administration) - (urinary marker before cisplatin administration). NGAL and L-FABP were measured only before cisplatin administration

University Medical and Dental Hospital. Urinary concentrations of Cr, NAG, $\alpha_{1}$-MG, and $\beta_{2}$-MG were measured with an automated instrument (7170S; Hitachi High-Technologies Corp., Tokyo, Japan) using the reagent kits CRE-S (Denka Seiken Co., Ltd., Gosen, Japan), N-assay L NAG NITTOBO (Nittobo Medical Co., Ltd.,
Tokyo, Japan), aMi-Latex (Denka Seiken Co., Ltd.), and BMG-Latex (Denka Seiken Co., Ltd.), respectively, at the laboratory of Denka Co., Ltd. Urinary NGAL was measured on the Abbott ARCHITECT i1000sr system at the laboratory of SRL Co., Ltd. (Tokyo, Japan). L-FABP was measured with a High Sensitivity Human L-FABP ELISA Kit (CMIC Holdings Co., Ltd., Tokyo, Japan) in our laboratory. The concentrations of each urinary marker were normalized to those of urinary $\mathrm{Cr}$ (/g Cr). eGFR was calculated using an equation that has been validated for the Japanese population [21]. $\triangle \mathrm{eGFR}$ and $\Delta$ urinary markers were calculated as follows: $\Delta$ eGFR $(\mathrm{mL} / \mathrm{min} /$ $\left.1.73 \mathrm{~m}^{2}\right)=($ minimum eGFR after cisplatin administration) - (eGFR before cisplatin administration); $\Delta$ urinary marker $=($ maximum urinary marker after cisplatin administration) - (urinary marker before cisplatin administration).

\section{Statistical analysis}

The subjects were classified into 4 groups according to quartile of baseline urinary A-megalin. Numerical data are expressed as the mean \pm standard deviation, and categorical data are expressed as $n$ (\%). Differences between groups were tested by one-way analysis of variance for numerical variables and the chi-square test for categorical variables. The correlation between two numerical variables was examined using Pearson's correlation coefficient $(r)$. Event-free survival curves were drawn using the Kaplan-Meier method, and differences between curves were tested by log-rank test. Mean differences in $\triangle \mathrm{eGFR}$ according to quartile of urinary A-megalin were estimated using crude and adjusted models of linear regression analysis with the lowest quartile (Q1) as the

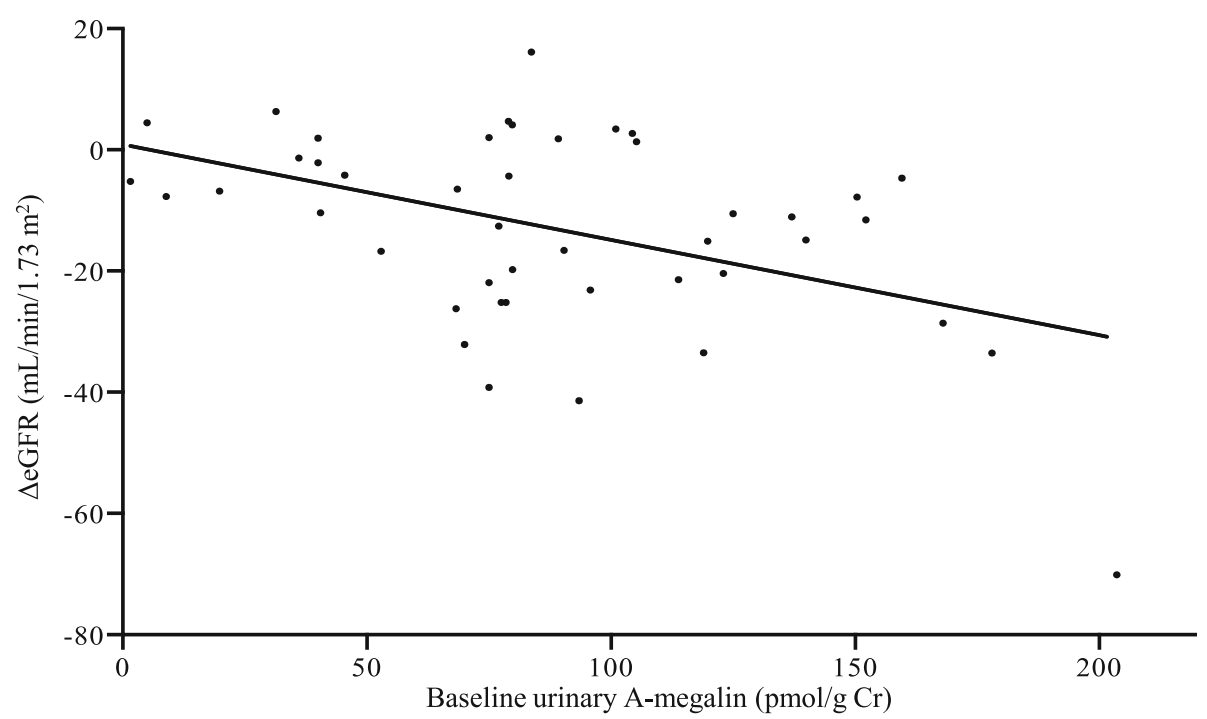

Fig. 2 Scatter diagram of the relationship between $\triangle$ eGFR and baseline urinary A-megalin levels. $\Delta e G F R\left(\mathrm{~mL} / \mathrm{min} / 1.73 \mathrm{~m}^{2}\right)=(\mathrm{minimum} \mathrm{eGFR}$ after cisplatin administration) - (eGFR before cisplatin administration). $r=-0.458, P=0.002$ 


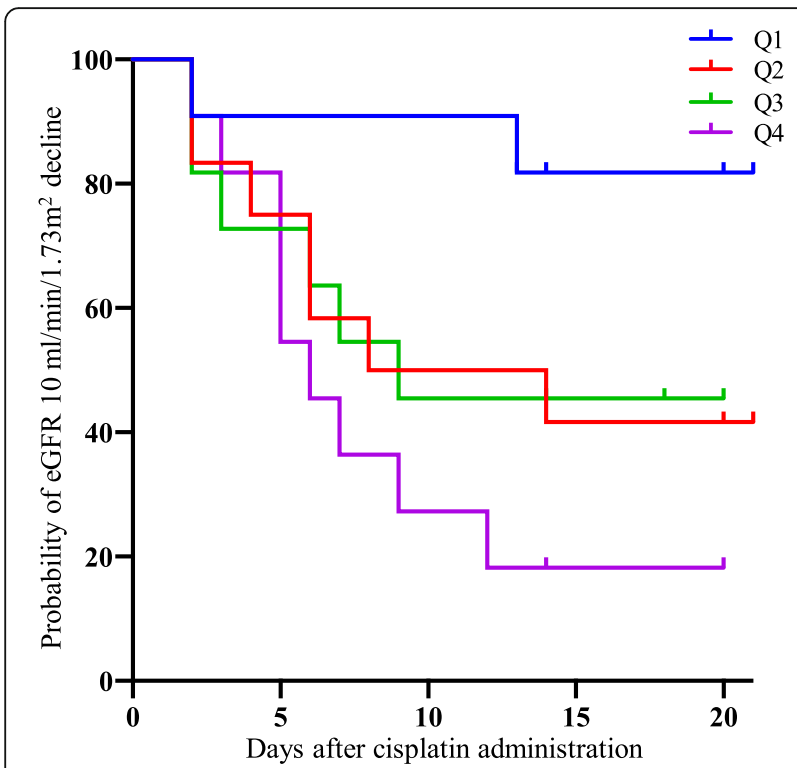

Fig. 3 Kaplan-Meier curves for adverse-renal-event-free survival. An adverse renal event was defined as eGFR decline $>10 \mathrm{~mL} / \mathrm{min} / 1.73$ $\mathrm{m}^{2}$. Tick marks indicate censored observations. Baseline higher urinary A-megalin levels tended to be associated with poorer adverse-renal-event-free survival $(P=0.038$, log-rank test)

reference. The adjusted model was adjusted for baseline eGFR. For these models, $P$-values for trend were also calculated using the quartile median values. Cox proportional hazards regression analysis was used to calculate crude and adjusted hazard ratios (HRs) and 95\% confidence intervals (CIs) for the first adverse renal event according to baseline urinary A-megalin quartiles. All statistical analysis was performed using SPSS v.18.0 (IBM Corp., Armonk, NY). The level of significance was two-tailed $P<0.05$.

\section{Results}

The baseline characteristics of the subjects according to quartile of baseline urinary A-megalin are shown in
Table 1. There were no significant differences between the groups in age, sex, smoking history, use of RAS inhibitors or NSAIDs, presence of hypertension or diabetes mellitus, baseline eGFR, and cisplatin dose. Tumor types were NSCLC $(n=33)$, SCLC $(n=10)$, and MPM $(n=2)$. Concomitant chemotherapeutic agents included pemetrexed $(n=18)$, etoposide $(n=12)$, gemcitabine $(n=9)$, CPT-11 $(n=3)$, docetaxel $(n=2)$, and vinorelbine $(n=1)$. Seven patients received the antivascular endothelial growth factor mAb bevacizumab with cisplatin and pemetrexed (Additional file 1: Table S1).

We previously reported that urinary A- and C-megalin levels in healthy control individuals were 73 (35-106) and $0.145(0.187-0.233) \mathrm{pmol} / \mathrm{g}$ Cr, respectively [14]. Mean baseline urinary A-megalin in the present study population $(87.9 \pm 46.6 \mathrm{pmol} / \mathrm{g} \mathrm{Cr})$ was nearly equal to that in our previous report, and mean baseline urinary $\mathrm{C}$-megalin in the present study $(0.64 \pm 0.76 \mathrm{pmol} / \mathrm{g} \mathrm{Cr})$ was slightly higher (Additional file 1: Table S2). Mean NAG, $\alpha_{1}-\mathrm{MG}, \beta_{2}-\mathrm{MG}, \mathrm{NGAL}$, and L-FABP are also shown in Additional file 1: Table S2.

During 564 person-days of follow-up, 24 cases (53.3\%) experienced adverse renal events (Fig. 1); the incidence rate of the first event was 0.426 per 10 person-days. Of those patients, the mean follow-up period until the first event was $5.8 \pm 3.5$ days from the start of cisplatin treatment. We found no association between pemetrexed administration and nephrotoxicity (data not shown).

According to Pearson's correlation coefficients, the baseline values of urinary A-megalin and eGFR showed negative correlations with $\Delta$ eGFR $(r=-0.458, P=0.002$ and $r=-0.395, P=0.007$, respectively): the correlation matrixes are shown in Table 2 and Additional file 1: Table S3, and the scatter diagram is shown in Fig. 2. In addition, A-megalin levels were not correlated with other urinary markers, including $\mathrm{C}$-megalin, at baseline. The other urinary markers did not show any correlation

Table 3 Difference in $\triangle \mathrm{eGFR}$ and the risk of adverse renal events according to quartile of urinary A-megalin

\begin{tabular}{|c|c|c|c|c|c|c|c|c|c|}
\hline \multirow[b]{3}{*}{$\Delta \mathrm{eGFR}\left(\mathrm{mL} / \mathrm{min} / 1.73 \mathrm{~m}^{2}\right)$} & \multicolumn{8}{|c|}{ Quartile of urinary A-megalin } & \multirow{3}{*}{$\begin{array}{l}P \\
0.085^{a}\end{array}$} \\
\hline & \multicolumn{2}{|c|}{$\mathrm{Q} 1(n=11)$} & \multicolumn{2}{|c|}{$\mathrm{Q} 2(n=12)$} & \multicolumn{2}{|c|}{$\mathrm{Q} 3(n=11)$} & \multicolumn{2}{|c|}{$\mathrm{Q} 4(n=11)$} & \\
\hline & -3.8 & \pm 6.7 & -15.2 & \pm 15.1 & -11.9 & \pm 18.0 & -20.8 & \pm 18.5 & \\
\hline \multicolumn{10}{|c|}{ Estimated difference (95\% Cl) from Q1 (mL/min/1.73 m²) } \\
\hline Crude & 0.00 & (reference) & -11.39 & $(-24.30,1.51)^{+}$ & -8.05 & $(-21.23,5.14)$ & -16.95 & $(-30.13,-3.76)^{*}$ & $0.018^{b}$ \\
\hline Adjusted & 0.00 & (reference) & -10.00 & $(-22.29,2.38)$ & -4.94 & $(-17.73,7.85)$ & -13.27 & $(-26.17,-0.37)^{*}$ & $0.064^{b}$ \\
\hline Adverse renal events / person-days & 2 & / 194 & 7 & / 146 & 6 & $/ 129$ & 9 & / 95 & \\
\hline Event rate (/10 person days) & 0.103 & & 0.479 & & 0.465 & & 0.947 & & \\
\hline \multicolumn{10}{|l|}{ Hazard ratio $(95 \% \mathrm{Cl})$} \\
\hline Crude & 1.00 & (reference) & 4.25 & $(0.88,20.51)^{+}$ & 4.04 & $(0.81,20.09)^{+}$ & 7.24 & $(1.55,33.96)^{*}$ & $0.008^{b}$ \\
\hline Adjusted & 1.00 & (reference) & 3.80 & $(0.78,18.48)^{+}$ & 3.03 & $(0.60,15.30)$ & 4.39 & $(0.91,21.13)^{+}$ & $0.093^{b}$ \\
\hline
\end{tabular}

$\mathrm{Cl}$, confidence interval; eGFR, estimated glomerular filtration rate. $\triangle$ eGFR, maximum change from the baseline value to the lowest value of eGFR during follow-up. An adverse renal event was defined as an eGFR decline of $>10 \mathrm{~mL} / \mathrm{min} / 1.73 \mathrm{~m}^{2}$. Adjusted hazard ratio, adjusted for baseline eGFR. ${ }^{+} P<0.1,{ }^{*} P<0.05,{ }^{a} P$ for statistical difference between groups, ${ }^{\mathrm{b}} P$ for trend 
with $\triangle \mathrm{eGFR}$. The adverse-renal-event-free KaplanMeier survival curves showed that higher baseline urinary A-megalin levels tended to be associated with poorer outcome ( $P=0.038$, log-rank test) (Fig. 3). By quartile of A-megalin, risk of eGFR decline was lowest in Q1, intermediate in Q2 and Q3, and highest in Q4.

When mean $\triangle \mathrm{eGFR}$ was compared between the quartiles of baseline urinary A-megalin, the decline in eGFR was greater in the highest quartile (Q4) than in the lowest quartile (Q1): the adjusted mean difference in $\triangle \mathrm{eGFR}$ between Q4 and Q1 was $-13.27 \mathrm{~mL} / \mathrm{min} / 1.73 \mathrm{~m}^{2}(95 \%$ CI, -26.168 to $-0.374, P=0.012$ ) (Table 3). The elevated risk of an adverse renal event associated with elevated A-megalin levels (Q4 vs. Q1) was significant in the crude model and marginally significant in the adjusted model (adjusted HR, 4.39; 95\% CI, 0.911 to 21.134, $P=0.065$ ).

\section{Discussion}

We examined the ectodomain (A-megalin) and full-length (C-megalin) forms of megalin in urine as markers of cisplatin-induced nephrotoxicity and found that prechemotherapy urinary A-megalin levels were associated with the development of cisplatin-induced nephrotoxicity. This is the first report to describe a relationship between prechemotherapy A-megalin levels and cisplatin-induced nephrotoxicity and to demonstrate that prechemotherapy A-megalin levels may be useful for predicting cisplatininduced nephrotoxicity.

We previously reported that urinary C-megalin levels are correlated with severity of diabetic kidney disease [14] and IgA nephropathy [22]. The mechanism underlying urinary $\mathrm{C}$-megalin excretion is associated with exocytosis, based on the chronic lysosomal protein metabolic load on PTECs of residual functioning nephrons [15]. However, baseline urinary $\mathrm{C}$-megalin, NAG, $\alpha_{1}-\mathrm{MG}, \beta_{2}-\mathrm{MG}$, NGAL, and L-FABP showed no correlation with the development of cisplatin-induced nephrotoxicity, suggesting that the mechanisms underlying urinary excretion of C-megalin and the other markers are not primarily associated with the pathogenesis of cisplatin-induced nephrotoxicity, at least in the patients enrolled in the present study.

In contrast, urinary A-megalin excretion was not correlated with other urinary markers including $\mathrm{C}$-megalin and appears to be regulated by a distinct mechanism. Megalin undergoes intracellular recycling in PTECs and metalloprotease-mediated ectodomain shedding by regulated intramembrane proteolysis $[17,18]$. Thus, urinary A-megalin may be produced as a normal byproduct in the absence of PTECs damage and may be increased by some factors that accelerate intracellular recycling. The efficiency of megalin recycling is also associated with its endocytic function [16]. Hence, it is likely that baseline urinary A-megalin is correlated with the amount and/or endocytic rate of megalin expressed in PTECs. It remains to be determined what factors are involved in the regulation of megalin recycling and/or endocytosis. We recently reported that megalin-mediated cisplatin uptake in PTECs has a primary role in nephrotoxicity [12]. Thus, baseline urinary A-megalin might reflect the efficiency of megalin-mediated cisplatin uptake in PTECs, and thus could be indicative of the development of cisplatin-induced nephrotoxicity. We also demonstrated that cilastatin, a megalin antagonist, blocks the binding of cisplatin to megalin [12], and thereby could protect against cisplatin-induced nephrotoxicity [23]. Hence, it would be worthwhile to investigate whether blockade of cisplatin binding to megalin could prevent cisplatininduced nephrotoxicity, particularly in patients with elevated urinary A-megalin levels.

The present study has several limitations. First, this was a single-institutional study with a limited number of patients. Further studies in different institutions and a large sample size are needed to verify our findings. Second, the relationship between urinary megalin levels and long-term renal dysfunction was not examined. Finally, we defined the adverse renal event as eGFR decline $>10$ $\mathrm{mL} / \mathrm{min} / 1.73 \mathrm{~m}^{2}$ [20]. In clinical practice, the Kidney Disease Improving Global Outcomes criteria are often used as a definition of acute kidney injury; however, there were few patients who met the criteria in this study.

\section{Conclusions}

This is the first report demonstrating that prechemotherapy urinary A-megalin levels are correlated with the development of cisplatin-induced nephrotoxicity and may be a novel predictor of nephrotoxicity. This has clinical implications for identifying patients at risk for cisplatin-induced nephrotoxicity and developing possible prophylactic therapies.

\section{Supplementary information}

Supplementary information accompanies this paper at https://doi.org/10. 1186/s12885-019-6398-2.

Additional file 1: Table S1. Chemotherapy regimens. Table S2. Baseline urinary markers. Table S3. Correlation matrix for all pairs of tested biomarkers.

\footnotetext{
Abbreviations

Cl: Confidence interval; $\mathrm{Cr}$ : Creatinine; eGFR: Estimate glomerular filtration rate; ELISA: Enzyme-linked immunosorbent assay; HR: Hazard ratio; LFABP: Liver-type fatty acid-binding protein; mAb: Monoclonal antibody; MEG: Megalin; MPM: Malignant pleural mesothelioma; NAG: N-acetyl- $\beta-D-$ glucosaminidase; NGAL: Neutrophil gelatinase-associated lipocalin; NSAID: Non-steroidal anti-inflammatory drug; NSCLC: Non-small cell lung cancer; PTECs: Proximal tubule epithelial cells; RAS: Renin-angiotensinaldosterone system; SCLC: Small cell lung cancer; SD: Standard deviation; $a_{1-}$ MG: $a_{1}$-microglobulin; $\beta_{2}-M G: \beta_{2}$-microglobulin
} 


\section{Acknowledgements}

We thank ThinkSCIENCE, Inc. for English editing of this manuscript.

\section{Authors' contributions}

HK2 and AS conceived and designed the experiments. SS, MH, and AS wrote the manuscript. HK1, RK2, SK, YH, and NT contributed to the analysis and interpretation of the data and assisted in the preparation of the manuscript. RK1, SM, SW, and NA assisted in the care of patients and collection of data. IN and TK provided administrative support. All authors read and approved the final manuscript.

\section{Funding}

This work was supported by Grants-in-Aid for Scientific Research to HK2 (no. 26293196) and AS (no. 26461216) and for Young Scientists (B) to NA (no. 25870245) from the Ministry of Education, Culture, Sports, Science and Technology of Japan, and by a Grant-in-Aid for the Practical Research Project for Renal Diseases from the Japan Agency for Medical Research and Development (18ek0310007h0003). The funding bodies had no role in the design of the study and collection, analysis, and interpretation of data and in writing the manuscript.

\section{Availability of data and materials}

The datasets used and/or analyzed during the current study are available from the corresponding authors on reasonable request.

\section{Ethics approval and consent to participate}

This study was approved by the Institutional Ethics Committee of Niigata University (approval number: 2015-1789). The study population included 45 patients who had given written informed consent.

\section{Consent for publication}

Not applicable.

\section{Competing interests}

AS received a research grant from Denka Seiken Co., Ltd. The other authors declare they have no competing interests.

\section{Author details}

Department of Respiratory Medicine and Infectious Diseases, Niigata University Graduate School of Medical and Dental Sciences, 1-757 Asahimachi-dori, Chuo-ku, Niigata, Niigata 951-8510, Japan. ${ }^{2}$ Department of Clinical Nutrition Science, Kidney Research Center, Niigata University Graduate School of Medical and Dental Sciences, 1-757 Asahimachi-dori, Chuo-ku, Niigata, Niigata 951-8510, Japan. ${ }^{3}$ Present address: Department of Internal Medicine, Niigata Cancer Center Hospital, 2-15-3 Kawagishi-cho Chuo-ku, Niigata, Niigata 951-8566, Japan. ${ }^{4}$ Department of Clinical Nephrology and Rheumatology, Kidney Research Center, Niigata University Graduate School of Medical and Dental Sciences, 1-757 Asahimachi-dori, Chuo-ku, Niigata, Niigata 951-8510, Japan. ${ }^{5}$ Department of Applied Molecular Medicine, Kidney Research Center, Niigata University Graduate School of Medical and Dental Sciences, 1-757 Asahimachi-dori, Chuo-ku, Niigata, Niigata 951-8510, Japan. ${ }^{6}$ Present address: Laboratory of Clinical Nutrition, Department of Nutrition, School of Human Cultures, The University of Shiga Prefecture, Hikone, Shiga, Japan. ${ }^{7}$ Department of Health and Nutrition, Faculty of Human Life Studies, University of Niigata Prefecture, 471 Ebigase, Higashi-ku, Niigata, Niigata 950-8680, Japan. ${ }^{8}$ Reagent Research and Development Department, Denka Seiken Co., Ltd., 1-2-3 Minamihoncho, Gosen, Niigata 959-1695, Japan. ${ }^{9}$ Present address: Division of Respiratory Medicine, Saitama Medical University International Medical Center, 1397-1 Yamane, Hidaka, Saitama 350-1298, Japan.

\section{Received: 12 September 2019 Accepted: 22 November 2019}

\section{8. - -10}

\section{References}

1. Lebwohl D, Canetta R. Clinical development of platinum complexes in cancer therapy: an historical perspective and an update. Eur J Cancer. 1998; 34(10):1522-34.

2. Siddik $\mathrm{ZH}$. Cisplatin: mode of cytotoxic action and molecular basis of resistance. Oncogene. 2003;22(47):7265-79.
3. Ries F, Klastersky J. Nephrotoxicity induced by cancer chemotherapy with special emphasis on cisplatin toxicity. Am J Kidney Dis. 1986;8(5):368-79.

4. Pabla N, Dong Z. Cisplatin nephrotoxicity: mechanisms and renoprotective strategies. Kidney Int. 2008:73(9):994-1007.

5. Ludwig T, Riethmüller C, Gekle M, Schwerdt G, Oberleithner $H$. Nephrotoxicity of platinum complexes is related to basolateral organic cation transport. Kidney Int. 2004;66(1):196-202.

6. Pabla N, Murphy RF, Liu K, Dong Z. The copper transporter Ctr1 contributes to cisplatin uptake by renal tubular cells during cisplatin nephrotoxicity. Am J Physiol Renal Physiol. 2009;296(3):F505-11.

7. Saito A, Pietromonaco S, Loo AK, Farquhar MG. Complete cloning and sequencing of rat gp330/"megalin," a distinctive member of the low density lipoprotein receptor gene family. Proc Natl Acad Sci U S A. 1994;91(21):9725-9.

8. Christensen El, Nielsen S, Moestrup SK, Borre C, Maunsbach AB, de Heer E, et al. Segmental distribution of the endocytosis receptor gp330 in renal proximal tubules. Eur J Cell Biol. 1995;66(4):349-64.

9. De S, Kuwahara S, Saito A. The endocytic receptor megalin and its associated proteins in proximal tubule epithelial cells. Membranes (Basel). 2014;4(3):333-55.

10. Kuwahara S, Hosojima M, Kaneko R, Aoki H, Nakano D, Sasagawa T, et al. Megalin-mediated Tubuloglomerular alterations in high-fat diet-induced kidney disease. J Am Soc Nephrol. 2016;27(7):1996-2008.

11. Moestrup SK, Cui S, Vorum H, Bregengård C, Bjørn SE, Norris K, et al. Evidence that epithelial glycoprotein 330/megalin mediates uptake of polybasic drugs. J Clin Invest. 1995:96(3):1404-13.

12. Hori Y, Aoki N, Kuwahara S, Hosojima M, Kaseda R, Goto S, et al. Megalin blockade with Cilastatin suppresses drug-induced nephrotoxicity. J Am Soc Nephrol. 2017;28(6):1783-91.

13. Suzuki T, Yamaguchi H, Ogura J, Kobayashi M, Yamada T, Iseki K. Megalin contributes to kidney accumulation and nephrotoxicity of colistin. Antimicrob Agents Chemother. 2013;57(12):6319-24.

14. Ogasawara S, Hosojima M, Kaseda R, Kabasawa H, Yamamoto-Kabasawa $\mathrm{K}$, Kurosawa $\mathrm{H}$, et al. Significance of urinary full-length and ectodomain forms of megalin in patients with type 2 diabetes. Diabetes Care. 2012; 35(5):1112-8

15. De S, Kuwahara S, Hosojima M, Ishikawa T, Kaseda R, Sarkar P, et al. Exocytosis-mediated urinary full-length Megalin excretion is linked with the pathogenesis of diabetic nephropathy. Diabetes. 2017:66(5):1391-404.

16. Suruda C, Tsuji S, Yamanouchi S, Kimata T, Huan NT, Kurosawa H, et al. Decreased urinary excretion of the ectodomain form of megalin (Amegalin) in children with OCRL gene mutations. Pediatr Nephrol. 2017; 32(4):621-5.

17. Zou Z, Chung B, Nguyen T, Mentone S, Thomson B, Biemesderfer D. Linking receptor-mediated endocytosis and cell signaling: evidence for regulated intramembrane proteolysis of megalin in proximal tubule. J Biol Chem. 2004;279(33):34302-10

18. Biemesderfer D. Regulated intramembrane proteolysis of megalin: linking urinary protein and gene regulation in proximal tubule? Kidney Int. 2006; 69(10):1717-21.

19. Shah M, Baterina OY, Taupin V, Farquhar MG. ARH directs megalin to the endocytic recycling compartment to regulate its proteolysis and gene expression. J Cell Biol. 2013;202(1):113-27.

20. Fatima N, Niaz K. Maseeh-Uz-Zaman, Kamal S, Hameed a. nephrotoxicity of CDDP assessed estimating glomerular filtration rate with 99mTc-DTPA plasma sample method. J Coll Physicians Surg Pak. 2010;20(2):98-101.

21. Matsuo S, Imai E, Horio M, Yasuda Y, Tomita K, Nitta K, et al. Revised equations for estimated GFR from serum creatinine in Japan. Am J Kidney Dis. 2009:53(6):982-92.

22. Seki T, Asanuma K, Asao R, Nonaka K, Sasaki Y, Oliva Trejo JA, et al. Significance of urinary full-length megalin in patients with IgA nephropathy. PLoS One. 2014;9(12):e114400.

23. Humanes B, Lazaro A, Camano S, Moreno-Gordaliza E, Lazaro JA, BlancoCodesido $\mathrm{M}$, et al. Cilastatin protects against cisplatin-induced nephrotoxicity without compromising its anticancer efficiency in rats. Kidney Int. 2012:82(6):652-63.

\section{Publisher's Note}

Springer Nature remains neutral with regard to jurisdictional claims in published maps and institutional affiliations. 